\title{
Características biológicas de una población de Pteria sterna (Bivalvia: Pteriidae) en Zorritos, Tumbes, Perú
}

\author{
Biological characteristics of one Pteria sterna population (Bivalvia: Pteriidae) from Zorritos, \\ Tumbes, Peru
}

\author{
Elmer Ordinola, Solange Alemán y Manuel Vera
}

\author{
IMARPE, Instituto del Mar del Perú - Laboratorio Costero \\ de Tumbes, Calle José Olaya S/N, C.P. Nueva Esperanza, \\ Zorritos, Contralmirante Villar, Tumbes. \\ Email Elmer Ordinola: elmord@imarpe.gob.pe \\ Email Solange Alemán: saleman@imarpe.gob.pe \\ Email Manuel Vera: mvera@imarpe.gob.pe
}

Citación:

Ordinola E., S. Alemán \& M. Vera. 2013. Características biológicas de una población de Pteria sterna (Bivalvia: Pteriidae) en Zorritos, Tumbes, Perú. Rev. peru. biol. 20(2) 181 - 186 (Diciembre 2013)

\begin{abstract}
Resumen
Entre abril y noviembre de 2011 se analizó una población de Pteria sterna (Gould, 1851) que se encontraba adherida a una red de cerco hundida en Zorritos, Tumbes, Perú ( $3^{\circ} 40^{\prime} 30.7^{\prime \prime} S$ - 8040'20.8”W). Los individuos presentaron tallas de 5 a $94 \mathrm{~mm}$ de altura valvar. La talla promedio se incrementó hacia finales del periodo evaluado. La estructura de tallas mostró la presencia de reclutas en la mayoría de meses evaluados, con máximos en abril y agosto. Se registraron cinco grupos de edad. La proporción sexual fue 1:1. Los desoves fueron continuos, a excepción de junio, con un máximo en noviembre. La relación altura valvar-peso total no mostró diferencias por sexos. El crecimiento de esta especie fue alométrico negativo. Las demás relaciones (potencial: peso del cuerpo-peso total, y lineales: peso del cuerpo-peso total y peso del talo - peso total) presentaron elevadas correlaciones. El rendimiento del músculo (talo) representó el $6.3 \%$ del peso total.
\end{abstract}

Palabras clave: Concha perlífera, tallas, desove, rendimiento, Zorritos, Perú.

\section{Abstract}

Between April and November 2011 was analyzed a population of Pteria sterna (Gould, 1851 ) which was attached to a sunken purse seine in Zorritos, Tumbes, Peru ( $3^{\circ} 40^{\prime} 30.7^{\prime \prime S}$ - $80^{\circ} 40^{\prime} 20.8^{\prime \prime W}$ ). Individuals presented from 5 to $94 \mathrm{~mm}$ valve height. The average size increased towards the end of the assessment period. The size structure indicated the presence of recruits in most months evaluated, with highest in April and August. There were five age groups. The sex ratio was 1:1. The spawns were continuous, except in June, with highest in November. Valvar height-total weight relationship did not differ by sex. The growth of this species was negative allometric. Other relationships (potential: body weight-total weight, and linear: body weight-total weight and muscle weight-total weight) showed high correlations. Muscle performance accounted for $6.3 \%$ of the total weight.

Keywords: Pearl shell, sizes, spawning, yield, Zorritos, Peru.

\section{Introducción}

Pteria sterna (Gould, 1851) llamada comúnmente concha perla, perlera o perlífera, es un bivalvo que habita en la zona infralitoral marina y en manglares (Álamo \& Valdivieso 1997), desde el nivel de la más baja marea hasta $23 \mathrm{~m}$ de profundidad (Mora 1990), adherida por medio de un biso a substratos duros (rocas, corales gorgónidos y estructuras metálicas sumergidas) o arena gruesa (Monteforte 2005, Ordinola et al. 2010a).

Se distribuye desde California (México) hasta Pimentel (Perú) (Álamo \& Valdivieso 1997), con poblaciones bien identificadas en la costa del Pacífico tropical y subtropical de América, desde el Golfo de California a Talara, Perú (Keen 1971, Arizpe 1992, Ordinola et al. 2010a). Excepcionalmente, luego del evento El Niño 1982-83 se han reportado ejemplares de P. sterna en las bahías de Ancón (Lima) e Independencia (Ica) en Perú, y Mejillones en Chile (Paredes et al. 1998, Díaz \& Ortlieb 1993).

Es una especie que puede sobrevivir en aguas muy turbias, soportando temperaturas menores a $18{ }^{\circ} \mathrm{C}$ y mayores a $32^{\circ} \mathrm{C}$, y salinidades menores a 34.5 ups y mayores a 37 ups, características que le confieren una gran diversidad de estrategias para permanecer en una zona o extender su distribución geográfica (Araya-Núnez et al. 1991, del RíoPortilla et al. 1992, Monteforte 2005). 


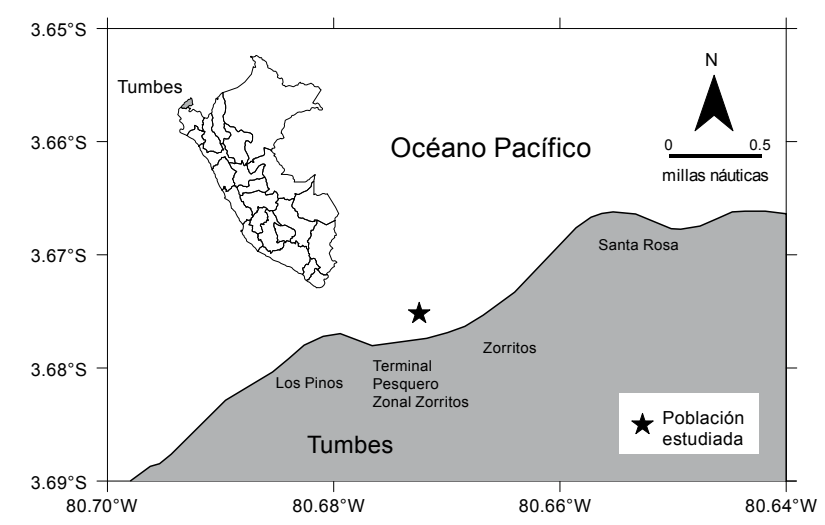

Figura 1.- Ubicación del banco de concha perlífera Pteria sterna (estrella) en Zorritos, Tumbes, Perú.

En Perú, las poblaciones naturales más importantes se encuentran en la provincia de Talara, Piura, específicamente frente a Punta Arenas, Negritos y Punta Capullanas, asociados a isotermas de 16.5 a $17{ }^{\circ} \mathrm{C}$, isohalinas de 35.1 a 35.15 ups y valores de oxígeno de $2 \mathrm{~mL} / \mathrm{L}$ (Ordinola et al. 2010a).

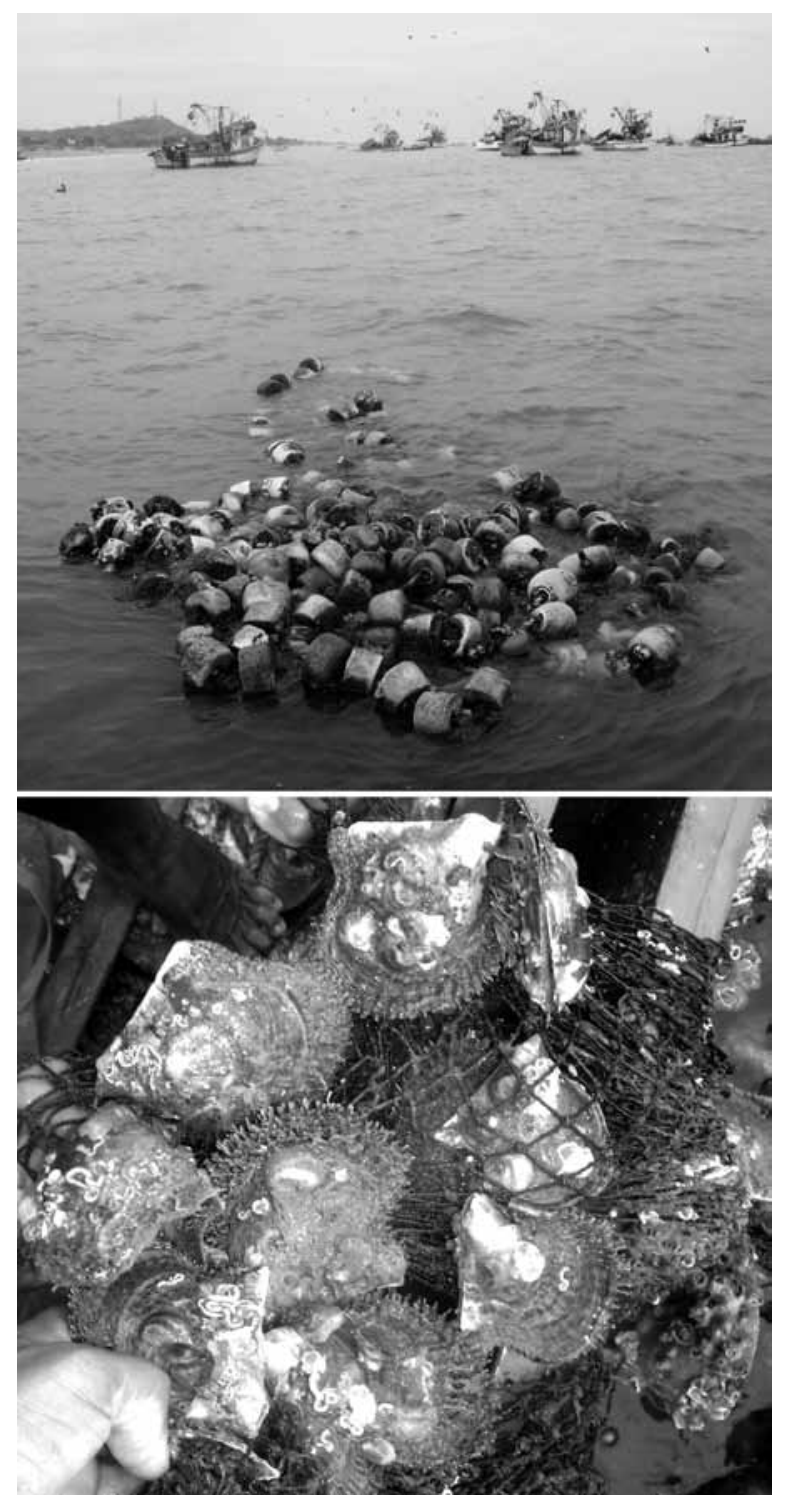

Figura 2.- Vista superior de la red de cerco hundida (A) y ejemplares de Pteria sterna adheridos a la misma (B) en Zorritos, Tumbes, Perú (Foto: S. Alemán).

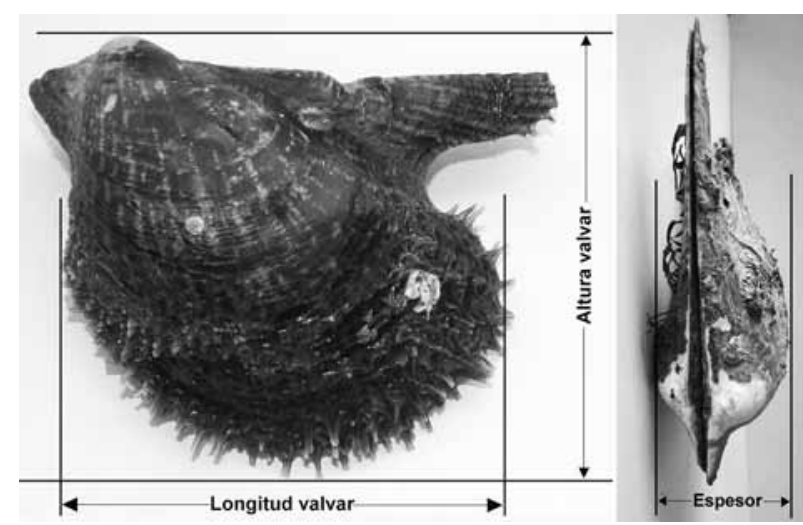

Figura 3.- Medidas morfológicas tomadas a los ejemplares de concha perlífera Pteria sterna (Foto: E. Ordinola).

En Tumbes, las poblaciones más importantes de concha perlífera están asentadas sobre las plataformas de extracción de petróleo ubicadas en La Cruz, Zorritos y Punta Sal, a profundidades de 3 a 45 m (Robles \& Méndez 1989).

El objetivo de este estudio fue caracterizar biológicamente la población de P. sterna asentada en una red de cerco atracada frente a Zorritos, Tumbes, Perú.

\section{Material y métodos}

Área de estudio.- El estudio se efectuó al noreste del desembarcadero artesanal de Zorritos (3०40'30,7”S - 8040'20,8”W) (Fig. 1). La población estudiada estaba asentada sobre una red de cerco de $38.1 \mathrm{~mm}$ de tamaño de malla, $1481.6 \mathrm{~m}$ de largo y $27.8 \mathrm{~m}$ de altura, atracada en el fondo a una profundidad de $4 \mathrm{~m}$, proveniente del naufragio de la embarcación LA BENDICION DE CRISTO PT-3439-CM [sic] (de 15 t de capacidad de bodega, $10.9 \mathrm{~m}$ de eslora, $4.1 \mathrm{~m}$ de manga y $1.81 \mathrm{~m}$ de puntal), ocurrido el 28 de enero de 2010.

Los muestreos iniciaron en abril de 2011 y siguieron hasta septiembre del mismo año. Los ejemplares se encontraban adheridos a la parte suspendida de la red en la columna de agua, que se disponía de forma envolvente, con la relinga superior expuesta en la superficie del mar (Fig. 2); en la parte de la red que estaba en contacto con el fondo no se encontraron ejemplares.

Colección de información y muestreo.- Los ejemplares fueron colectados por buceo libre. Se identificaron siguiendo a Keen (1971), Mora (1990) y Álamo y Valdivieso (1997), y se midieron en su longitud (LV), altura (AV) y espesor valvar (EV), con un malacómetro (graduado en $\mathrm{mm}$ ), según las técnicas descritas por Laevastu (1971), Fischer et al. (1995) y Galdámez et al. (2007) (Fig. 3). Además, se registró el peso total (PT), peso del cuerpo sin valvas (Pc) y peso del músculo o "talo" (Pm) de cada ejemplar, utilizando una balanza de $0.01 \mathrm{~g}$ de precisión.

Pteria sterna presenta sexos separados, que a simple vista no pueden ser identificados. Para determinar el sexo y la madurez gonadal solo en hembras, se empleo la siguiente metodologia: en primer lugar se efectuo un corte transversal entre la union del pie y la masa visceral, luego se extrajo una porcion de la masa gonadal, se coloco en una lamina portaobjeto y se ańadio una gota de agua cubriendose inmediatamente con una laminilla cubreobjeto, luego se observo al microspcopio y se determino el sexo, los machos fueron identificados por el movimiento de los espermatocitos y en hembras segun el 
grado de desarrollo de los ovocitos y trabeculas, se empleo la siguiente escala:

I. Inmaduro. Se observan trabéculas (especie de saco) que contienen en su parte interna numerosas células germinales que darán lugar a los ovocitos. En las paredes de estas trabéculas se observan ovogonias y/o ovocitos inmaduros pequeños.

II. En Maduracion. Las trabéculas son más grandes. Se observa la presencia de ovocitos más desarrollados.

III. Maduro. Las trabéculas son más grandes y de pared delgada. Numerosos ovocitos de forma redondeada o piriforme.

IV. En Evacuacion. Las trabéculas de menor tamaño. Pocos ovocitos maduros. Puede observarse ovocitos en proceso de reabsorción (atresia).

V. Post-Desove (o en recuperacion).Trabéculas de pared bastante gruesa, completamente vacías.

Análisis de datos.- Se obtuvieron los estadísticos para caracterizar la estructura de tallas de la especie mediante la variable AV (promedio, rango, moda y desviación estándar). Se aplicó el ANOVA de una vía y prueba de Tukey para determinar diferencias entre los valores mensuales de la altura valvar.

Se determinaron los grupos modales mediante descomposición de frecuencias de $\mathrm{AV}$, usando el método de Bhattacharya de la subrutina homónima del programa FiSAT II (Gayanilo et al.
2003). Las medidas de AV fueron agrupadas en clases de $5 \mathrm{~mm}$.

Se determinó la proporción sexual (PS) por meses, mediante la fórmula: PS = No machos / No hembras. Para determinar si existen diferencias estadísticas $(\mathrm{p}<0.05)$ se aplicó la prueba de Chi-cuadrado ( $a=0.05)$ (Zar 1999).

Se determinó el porcentaje mensual de ejemplares hembra según estadios de madurez gonadal.

Se analizaron las relaciones AV-PT y AV-Pm, utilizando las ecuaciones de potencia $\mathrm{PT}=\mathrm{a}(\mathrm{AV})^{\mathrm{b}}$ y $\mathrm{Pm}=\mathrm{a}(\mathrm{AV})^{\mathrm{b}}$, respectivamente, ajustadas por el método de mínimos cuadrados; y PT-Pc, empleando la ecuación lineal $\mathrm{Pc}=\mathrm{a}+\mathrm{b}(\mathrm{PT})$. Además se determinó el rendimiento del músculo $(\mathrm{Pm})$ en relación al PT de cada ejemplar analizado, empleando la ecuación lineal $\mathrm{Pm}=\mathrm{a}+\mathrm{b}(\mathrm{PT})$. En estas ecuaciones, a y b son constantes de ajuste.

Para determinar diferencias por sexo en la relación AV-PT $(\mathrm{p}<0.05)$, se aplicó el ANCOVA. Además se compararon las pendientes estimadas con la prueba $\mathrm{t}-$ Student de dos colas $(\mathrm{p}<$ 0.05 ), para evaluar el valor teórico de isometría, $b=3$ (Sokal \& Rohlf 1969).

\section{Resultados}

Estructura de tallas.- Entre abril y noviembre de 2011, se colectaron 622 ejemplares de concha perlífera (promedio de 89 ejemplares por mes), con rango de talla de 5 a $94 \mathrm{~mm} \mathrm{AV}$. La talla media se incrementó de $50.6 \mathrm{~mm}$ AV en abril a 65.4
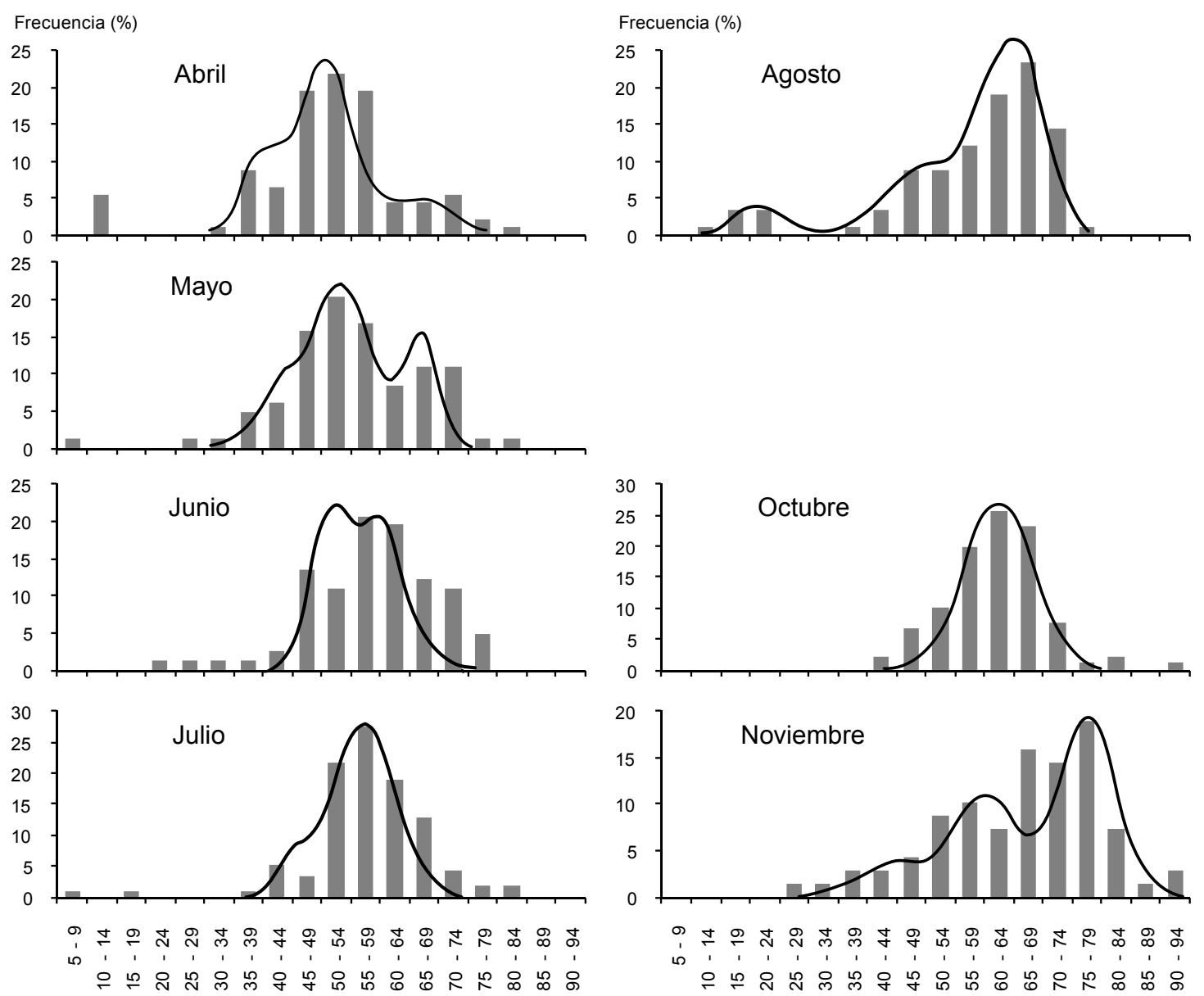

Figura 5.- Estructura de tallas y grupos modales por meses de concha perlífera Pteria sterna, en Zorritos, Tumbes, Perú (abril - noviembre 2011). 
Tabla 1.- Estadísticos biométricos por meses de concha perlífera Pteria sterna, en Zorritos, Tumbes, Perú (Abril - Noviembre 2011).

\begin{tabular}{lcccccc}
\hline \multirow{2}{*}{ Mes } & \multirow{2}{*}{$\mathbf{n}$} & \multicolumn{3}{c}{ Altura valvar $(\mathbf{m m})$} & \multirow{2}{*}{ DS } & \multirow{2}{*}{ Varianza } \\
\cline { 3 - 5 } & & Rango & Media & Moda & & \\
\hline Abril & 92 & $12-81$ & 50.6 & 58 & 13.7 & 187.4 \\
Mayo & 83 & $7-81$ & 54.6 & 57 & 12 & 144 \\
Junio & 82 & $20-79$ & 57.8 & 67 & 11 & 121.4 \\
Julio & 116 & $5-83$ & 57.1 & 55 & 10.3 & 105.2 \\
Agosto & 90 & $14-75$ & 57.6 & 65 & 14.4 & 207.1 \\
Octubre & 90 & $43-90$ & 61.3 & 61 & 8.6 & 74.6 \\
Noviembre & 69 & $28-94$ & 65.4 & 75 & 14 & 195.6 \\
$\quad$ Total & 622 & $5-94$ & 57.5 & 74 & 12.7 & 160.7 \\
\hline
\end{tabular}

n: número de ejemplares, DS: desviación estándar.

$\mathrm{mm}$ AV en noviembre, mostrando una variación de $14.8 \mathrm{~mm}$ durante los siete meses evaluados (+29.2\%) (Tabla 1). Se observó una tendencia positiva de la media durante abril a junio, para estabilizarse hasta agosto (invierno), e incrementar nuevamente de octubre a noviembre (Fig. 4). La estructura de tallas indicó la presencia de reclutamientos continuos con máximos en abril y agosto (Fig. 5).

Grupos modales.- Se diferenciaron como máximo tres grupos modales en abril, mayo, agosto y noviembre (la media \pm desviación

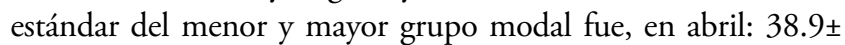
$3.25 \mathrm{~cm}$ AV y $70.7 \pm 6.85 \mathrm{~cm}$ AV; mayo: $40.5 \pm 4.64 \mathrm{~mm}$ AV y $70.0 \pm 3,21 \mathrm{~mm} \mathrm{AV}$; agosto: $19.5 \pm 4.77$ y $65.2 \pm 4.67 \mathrm{~mm} \mathrm{AV}$; y noviembre: $39.5 \pm 6.01 \mathrm{~mm}$ AV y $75.5 \pm 5.13 \mathrm{~mm} \mathrm{AV).} \mathrm{En} \mathrm{total}$ se detectaron cinco grupos de edad. El grupo 1 se presentó en agosto $(19.5 \pm 4.77 \mathrm{~mm} \mathrm{AV})$ y noviembre $(39.5 \pm 6.01 \mathrm{~mm} \mathrm{AV})$, el grupo 2 de julio $(43.6 \pm 3.37 \mathrm{~mm} \mathrm{AV})$ a noviembre $(56.7 \pm 5.98$ $\mathrm{mm}$ AV), el grupo 3 de abril $(38.9 \pm 3.25 \mathrm{~mm} \mathrm{AV})$ a noviembre $(75.5 \pm 5.13 \mathrm{~mm} \mathrm{AV})$, el grupo 4 de abril $(51.5 \pm 5.51 \mathrm{~mm} \mathrm{AV})$ a junio $(59.8 \pm 7.12 \mathrm{~mm} \mathrm{AV})$, y el grupo 5 en abril $(70.7 \pm 6.85$

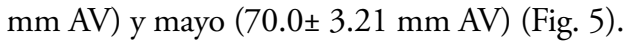

Proporción sexual.- Se analizaron 522 ejemplares de concha perlífera, de los cuales 162 fueron machos, 164 hembras, y 196 indeterminados. La proporción sexual global registrada (una hembra por cada macho) no se diferenció de la proporción esperada ( $\left.1 \mathrm{H}: 1 \mathrm{M} ; X^{2}=0.012 ; \mathrm{gl}=1 ; \mathrm{p}>0.05\right)$, aunque sólo en julio favoreció a los machos $\left(2 \mathrm{M}: 1 \mathrm{H} ; X^{2}=5.0\right.$; $\mathrm{gl}=1$; $\mathrm{p}<$ 0.05) (Fig. 6).

Madurez gonadal.- En las 156 hembras analizadas, predo-

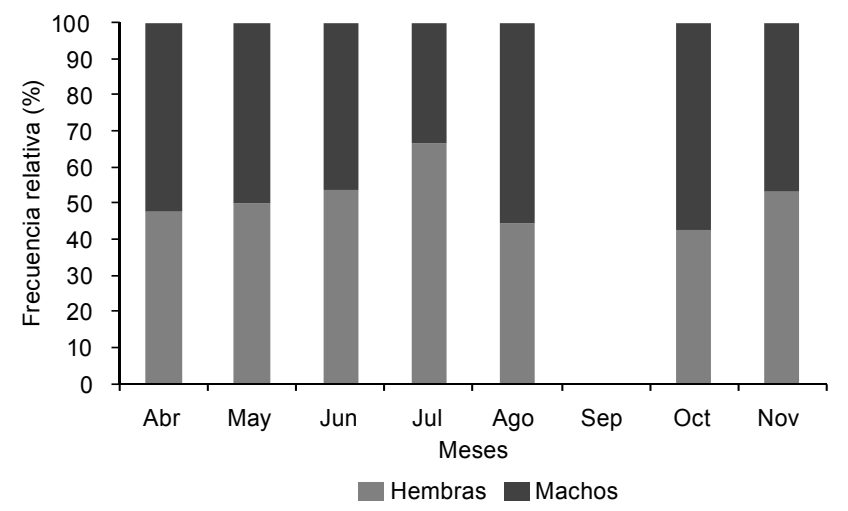

Figura 6.- Proporción sexual por meses de concha perlifera Pteria sterna, en Zorritos, Tumbes, Perú (abril - noviembre 2011).

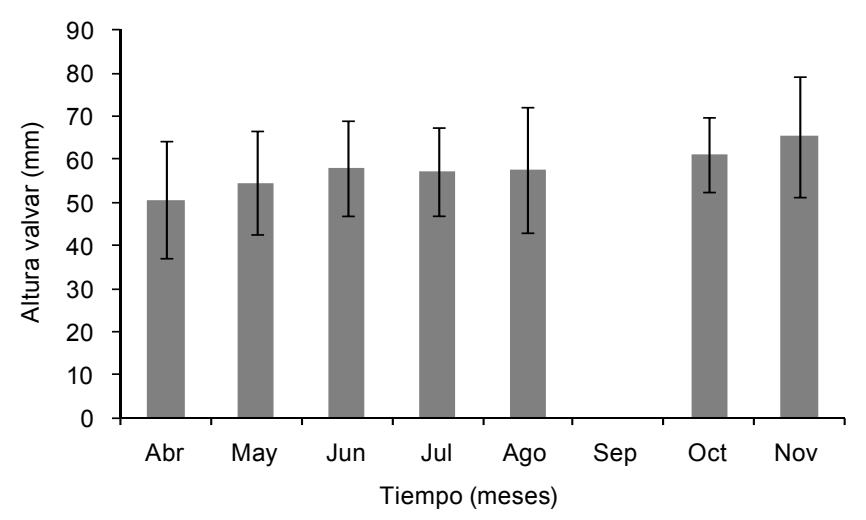

Figura 4.- Variación mensual de la altura valvar (AV, $\mathrm{mm}$ ), y desviación estándar, de concha perlífera Pteria sterna, en Zorritos, Tumbes, Perú (Abril - Noviembre 2011).

minaron los ejemplares maduros en abril, mayo, junio, agosto y octubre. Los mayores porcentajes en evacuación o desove se registraron en la mayoría de meses evaluados a excepción de junio, siendo noviembre el mes que más destacó con el 55.6\%. Hembras en post-desove fueron registradas durante el segundo semestre del año (julio, agosto, octubre y noviembre) (Fig. 7).

Relaciones biométricas.- Las ecuaciones de la relación AV-PT según sexos no presentaron diferencias significativas $(\mathrm{F}=$ $0.47 ; \mathrm{p}=0.4913)$ y mostraron elevadas correlaciones $(r>0.91)$, siendo la ecuación general para el período evaluado:

$\mathrm{PT}=8.543 \times 10^{-4}(\mathrm{AV})^{2.629}(\mathrm{n}=522 ; \mathrm{r}=0.9604)$ (Fig. 8A).

Las pendientes de la relación AV-PT, tanto por sexo (2.521 en machos y 2.673 en hembras) como para el total de la muestra (2.629), presentaron una relación alométrica negativa con respecto a la pendiente hipotética de isometría de $3\left(\mathrm{t}_{\mathrm{c}}>\mathrm{t}_{\mathrm{t}} ; \mathrm{p}<0.05\right)$.

La relación AV-Pm estuvo definida por la ecuación:

$\mathrm{Pm}=1.764 \times 10^{-5}(\mathrm{AV})^{2.879}(\mathrm{n}=511 ; \mathrm{r}=0.8711)$ (Fig. 8B).

La relación Pc-PT también mostró alta correlación ( $\mathrm{r}=$ 0,9319). La ecuación indicó que por cada $100 \mathrm{~g}$ de peso total se obtuvieron $27.3 \mathrm{~g}$ de peso de cuerpo (Fig. 9A).

Rendimiento.- El rendimiento del músculo, que es la parte que se comercializa en este molusco, representó el $6.3 \%$ del peso total, es decir que por cada $100 \mathrm{~g}$ de peso total se obtendrían $6.3 \mathrm{~g}$ de talo (Fig. 9B).

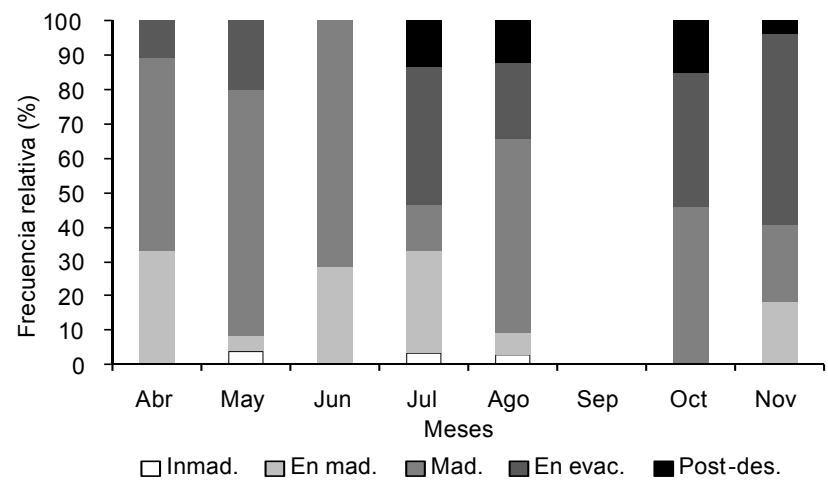

Figura 7.- Variación mensual de la madurez gonádica de hembras de concha perlífera Pteria sterna, en Zorritos, Tumbes, Perú (abril - noviembre 2011). 

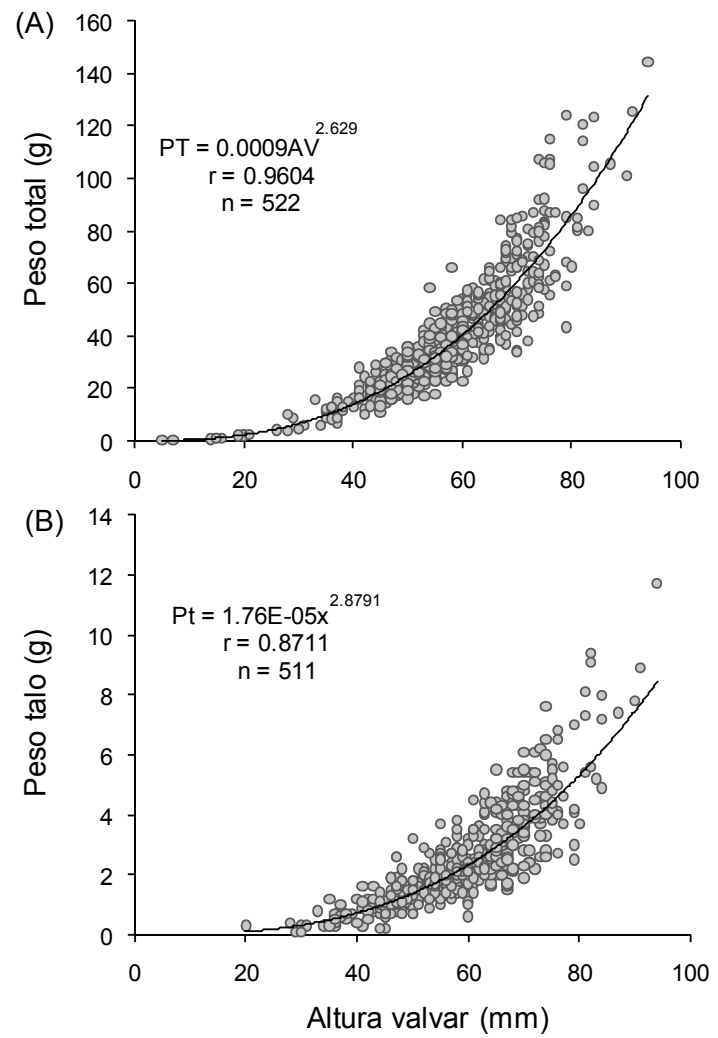

Figura 8.- Relaciones: A) altura valvar - peso total, y B) altura valvar peso músculo, de concha perlífera Pteria sterna, en Zorritos, Tumbes, Perú (Abril - Noviembre 2011).

\section{Discusión}

Se conoce que las poblaciones más importantes de concha perlífera en el litoral de Tumbes están asentados en las plataformas de extracción de petróleo ubicados a más de $3 \mathrm{mn}$ de las costas de caleta La Cruz, Zorritos y Punta Sal, a profundidades de 3 a 45 m, y cuyas densidades varían de 1 a 120 ejemplares. ${ }^{-2}$ (Robles \& Méndez 1989).

La población analizada en este estudio se asentó en una red de cerco hundida en Zorritos que presentó las características idóneas para su fijación. Al respecto, Araya-Núñez et al. (1991), del Río-Portilla et al. (1992) y Monteforte (2005) señalan que debido a la tendencia oportunista de esta especie y a su tolerancia a variaciones ambientales agresivas, despliega una gran diversidad de estrategias para asegurar su permanencia. Sin embargo, la continuidad de esta población dependerá del tiempo que demande a los pescadores locales extraer toda la relinga superior (flotadores), así como la misma red, lo que está ocurriendo actualmente.

La población se caracterizó por presentar ejemplares jóvenes (promedios mensuales de 50.6 mm AV a 65.4 mm AV), lo que de acuerdo a Monteforte (2005) es característico de una población en desarrollo. Las tallas medias encontradas fueron similares a las registradas por Robles y Méndez (1989), en la plataforma de petróleo de La Cruz (56 mm AV), por Kameya et al. (1996), para el litoral de Tumbes ( 32 y $67.7 \mathrm{~mm} \mathrm{AV}$ ) y por Ordinola et al. (2010b), frente a Canoas, Cancas y Punta Sal (51 mm AV), pero diferentes a la encontrada por Robles y Méndez (1989) en la plataforma de Zorritos (115 mm AV), población que ellos consideraron en su máximo desarrollo.
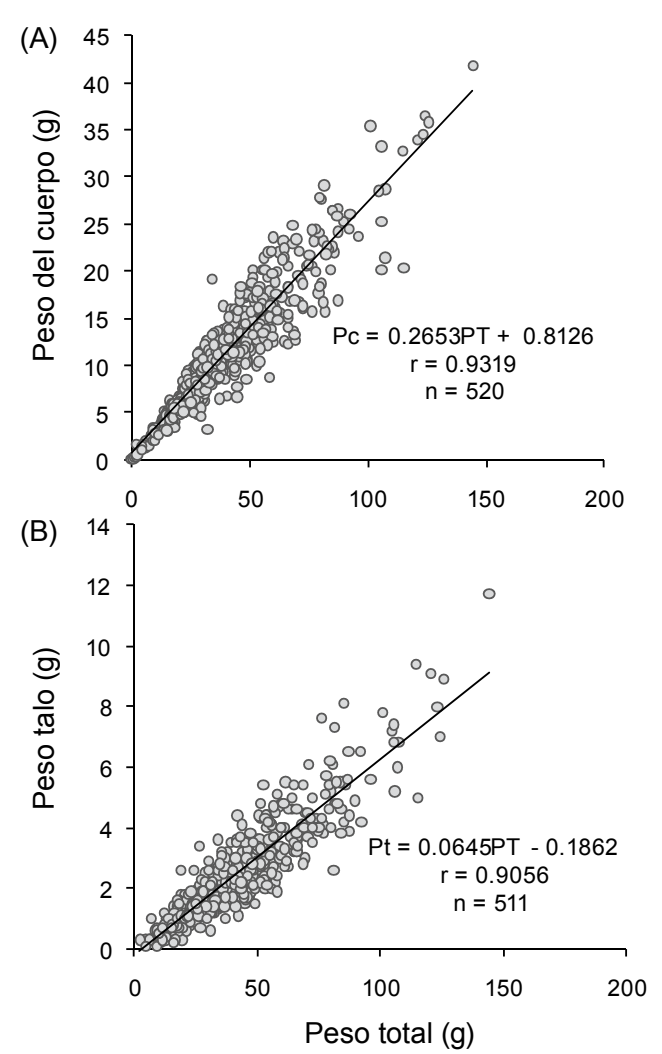

Figura 9.- Relaciones: A) peso total - peso cuerpo, y B) peso total - peso músculo (rendimiento), de concha perlífera Pteria sterna, en Zorritos, Tumbes, Perú (Abril - Noviembre 2011).

Aunque la formación de esta población es reciente, se detectaron cinco grupos de edad, similar a lo señalado por Kameya et al. (1996) y Robles y Méndez (1989), quienes registraron varias clases anuales durante estudios efectuados en 1995 y 1985, respectivamente. Sin embargo, el método empleado no habría registrado los grupos modales más pequeños durante abril a julio, posiblemente por la similitud de las tallas y la poca cantidad de ejemplares.

En la mayoría de meses evaluados se registraron ejemplares jóvenes, observándose el mayor ingreso de reclutas durante abril y agosto. Al respecto, Ruiz y Cáceres (1990) indican que en la Bahía de la Paz, Pacífico mexicano, P. sterna muestra reclutamientos en colectores artificiales durante todo el ańo, coincidiendo con la estrategia de reproducción continua, por lo que Márquez et al. (2000) sugieren que los factores ambientales no juegan un papel significativo en la regulación del ciclo reproductivo de esta especie y probablemente factores endógenos se encargan de su modulación, y recomiendan realizar estudios exhaustivos para verificar esta hipótesis.

La proporción sexual encontrada $(1 \mathrm{H}: 1 \mathrm{M})$ fue similar a la registrada por Díaz y Bückle-Ramírez (1996) en Baja California, México (1.2 H: 1 M), aunque diferente a la obtenida por Monteforte (2005) en ejemplares en cultivo (1 M: $0.38 \mathrm{H})$.

Se registraron desoves durante seis de los siete meses estudiados. Al respecto, Díaz y Bückle-Ramírez (1996) observaron que P. sterna realizó desoves parciales prácticamente todo el año, lo que fue confirmado por Monteforte (2005), quien registró la actividad reproductiva continua de esta especie en Bahía de La Paz, México, observando dos ciclos de desove y reclutamiento en colectores durante verano e invierno, aspecto que se observaría en 
las especies perleras netamente tropicales como Pinctada albina (Lamarck, 1819), P. margaritifera (Linnaeus, 1758), P. maxima (Jameson, 1901) y $P$. fucata (Gould, 1850).

El rendimiento del músculo (talo) (6.3\%) fue inferior al reportado por Kameya et al. (1996) en Tumbes (10\%), y Robles y Méndez (1989) en Talara (10\%) y Tumbes (8 a 11\%). Luque et al. (2001) obtuvieron en Talara rendimientos similares a los observados en el presente estudio ( $4.3 \%$ a $7.2 \%)$.

Con respecto a la relación $\mathrm{AV}-\mathrm{Pm}$, los pesos estimados fueron superiores a los obtenidos por Luque et al. (2001) y Ordinola et al. (2010a), lo que indicaría que la especie encontró condiciones aptas para su desarrollo.

Por lo tanto, la elevada presencia de ejemplares jóvenes indicaría que la población estudiada de $P$. sterna se encontraba en desarrollo.

\section{Agradecimientos}

Al Dr. Jorge Llanos, por las facilidades brindadas para la realización del presente trabajo, al Téc. William Coronado, por su apoyo en los muestreos biológicos, al Dr. Mario MonteforteSánchez, por proporcionar información relevante sobre la biología de P. sterna, así como por sus comentarios y recomendaciones, y al revisor anónimo, por sus correcciones y sugerencias que permitieron mejorar la redacción de este artículo.

\section{Literatura citada}

Álamo V. \& V. Valdivieso. 1997. Lista sistemática de moluscos marinos del Perú. Segunda Edición. Instituto del Mar del Perú. Callao - Perú. 183 pp.

Araya-Nuñez O., B. Ganning \& L.F. Bückle-Ramirez. 1991. Gonad maturity, induction of spawning, larval breeding and growth in the American pearl oyster (Pteria sterna, Gould). Calif. Fish. and Game, 77(4): 181-193.

Arizpe C.O. 1992. Los moluscos y su importancia comercial en el Pacífico mexicano. Serie Didáctica U.A.B.C.S., Num.1, 219 pp.

Arreola J. 1997. Aspectos reproductivos de Dosinia ponderosa, Gray 1838 (Bivalvia: Veneridae) en Punta Arena, Bahía Concepción, B.C.S. Instituto Politécnico Nacional. Centro Interdisciplinario de Ciencias Marinas. Tesis para obtener el Grado de Maestro en Ciencias con especialidad en Manejo de Recursos Marinos. La Paz - México. 85 pp.

Chirichigno N. 1970. Lista de crustáceos del Perú (Decapoda y Stomatopoda) con datos de su distribución geográfica. Inf. Inst. Mar Perú. 35. Callao - Perú. 95 pp.

Del Río-Portilla M.A., A.D. Re-Arayo \& D. Voltolina. 1992. Growth of the pearl oyster Pteria sterna under different thermic and feeding conditions. Mar. Ecol. Prog. Ser., 89: 221-227.

Díaz A.H. \& L.F. Bückle-Ramírez. 1996. Ciclo gonádico de Pteria sterna (Gould, 1851) (Mollusca: Bivalvia) en Baja California, México. Ciencias Marinas. Vol 22. Universidad Autónoma de Baja California. Ensenada - México. p: 495-509.

Díaz A. \& L. Ortlieb. 1993. El fenómeno "El Niño" y los moluscos de la costa peruana. Bull. Inst. fr. études andines. 22 (1): 159 - 177.

Fischer W., F. Krupp, W. Schneider, et al. 1995. Guía FAO para la identificación de especies para los fines de la pesca. Pacífico Centro Oriental. Volumen I: Plantas e Invertebrados. Sección: Cangrejos. FAO. Roma.
Galdámez A., S. Pacheco, I. Pérez \& S. Kino. 2007. Guía para la producción de Anadara spp. 2006 - 2007. Producción artificial de semillas, cultivo intermedio y cultivo de Anadara tuberculosa y A. grandis. Proyecto de Moluscos. CENDEPESCA - JICA. San Salvador. El Salvador.42 pp.

Gayanilo F.C., P. Sparre \& D. Pauly. 2003. FAO-ICLARM Stock Assessment Tools (FiSAT II) User's Guide (Rev. 1). FAO Computerized Information Series (Fisheries) No 8. Rome, FAO. 176 pp.

Keen A.M. 1971. Sea shells of tropical west America. Marine mollusks form Baja California to Peru. 2nd. Edition. Stanford University Press. Stanford. California. 1064 pp.

Laevastu T. 1971. Manual de métodos de biología pesquera. FAO. Editorial Acribia. Zaragoza - España. 243 pp.

Luque C., J. Solís, O. Morón, M. Crispín. 2001. Prospección de "concha perlífera” Pteria sterna en Talara, noviembre 2000. Inf. Prog. Inst. Mar Perú. No 154. Callao - Perú. 19 pp.

Márquez B., C. Lodeiros, M. Jiménez \& J.H. Himmelman. 2000. Disponibilidad de juveniles por captación natural de la ostra Pteria colymbus (Bivalvia: Pteriidae) en el Golfo de Cariaco, Venezuela. Rev. Biol. Trop. 48 Supl. 1:151-158.

Monteforte M. 2005. Ecología, biología y cultivo extensivo de la madreperla de calafia, Pinctada mazatlanica (Hanley 1856), y la concha nácar arcoíris Pteria sterna (Gould 1852) en Bahía de La Paz, Baja California Sur, México. Tesis para optar el grado de Doctor en Ciencias Biológicas. Universidad de La Habana. Cuba. 132 pp.

Mora E. 1990. Catálogo de bivalvos marinos del Ecuador. Instituto Nacional de Pesca. Boletín Científico Técnico. Vol. X. No 1. Guayaquil Ecuador. 136 pp.

Ordinola E., P. Montero, S. Alemán \& J Llanos. 2010a. El bivalvo concha perlífera Pteria sterna (Gould) en Talara, Perú. Abril 2007. Inf. Inst. Mar Perú. 37(3-4): 127-137.

Ordinola E., E. López, I. Gonzales, et al. 2010b. Delimitación y caracterización de bancos naturales de invertebrados bentónicos comerciales y áreas de pesca artesanal en el litoral de Tumbes. [Informe Interno Instituto del Mar del Perú] 79 pp. <http://www.imarpe.gob.pe/tumbes/noticias/Ident_delimit_B_N_Z_12-04-2010.pdf> Acceso 24/10/2012.

Paredes C., J. Tarazona, E. Canahuire, et al. 1998. Presencia de moluscos tropicales de la Provincia Panameńa en la costa central del Perú y su relación con los eventos "El Nińo". Rev. Perú. Biol. 5 (2):123-128.

Robles A. \& M. Méndez. 1989. Moluscos comerciales del litoral de Tumbes y Piura. Boletín de Lima. No 63. p: $47-70$.

Ruiz C.A. \& C.M. Cáceres. 1990. Estudio preliminar de captación de juveniles de moluscos bivalvos en la Bahía de la Paz, B.C.S. México. Inv. Mar. CICIMAR. 5(1):29-38.

Seed R. 1976. Ecology. In: B.L. Bayne, ed., Marine mussels their ecology and physiology. International Biological Program, Vol. 10. Cambridge University Press, Cambridge. p: 13-65.

Seed R. 1980. Shell growth and form in the Bivalvia. In: D.C. Rhoads, ed., Skeletal growth of aquatic organisms. Biological Records of Environmental Change. Plenum Press, New York. p: 23-61.

Sokal R.R. \& F.J. Rholf. 1969. Biometry. WH Freeman, San Francisco. 776 pp.

Wright-López H. 1997. Ecología de la captación de la semilla de madreperla Pinctada mazatlanica y concha nácar Pteria sterna (Bivalvia: Pteridae), en la Isla Gaviota, Bahía de La Paz, B.C.S., México. Tesis para optar el grado de Maestro en Ciencias. Instituto Politécnico Nacional. México. 138 pp.

Wright-López H., O. Holguín-Quiñones, F. Arreguín-Sánchez, I. RoqueVillada. 2009. Crecimiento y mortalidad de la concha nácar Pteria sterna en bancos silvestres de Baja California Sur, México. Rev. Biol. Trop. Vol 57 (3). p: $659-670$.

Zar J.H. 1999. Biostatistical analysis. 4th Edition. Prentice-Hall, Inc. N.J. 770 pp. 